\title{
MicroRNA-451 increases vascular permeability and suppresses angiogenesis in pulmonary burn injury in a rat model
}

\author{
${ }^{*}$ Jie Zhou ${ }^{1, B, D}$, *Huibin Lian ${ }^{2, B, C}$, Gang Xu ${ }^{1, A, F}$, Tianlan Zhao ${ }^{1, B, C}$ \\ ${ }^{1}$ Department of Plastic and Cosmetic Surgery, Second Affiliated Hospital of Soochow University, China \\ 2 Burns and Plastic Surgery, Northern Jiangsu People's Hospital, Yangzhou, China \\ A - research concept and design; $B$ - collection and/or assembly of data; $C$ - data analysis and interpretation; \\ $D$ - writing the article; $E$ - critical revision of the article; $F$ - final approval of the article
}

\section{Address for correspondence \\ Tianlan Zhao \\ E-mail:zt11965@163.com \\ Funding sources \\ The hospital-level Project of Northern Jiangsu People's Hospital (project No. yzucms201322). \\ Conflict of interest \\ None declared \\ * Jie Zhou and Huibin Lian contributed equally to this work.}

Received on February 17, 2020

Reviewed on June 8, 2020

Accepted on August 11, 2020

\begin{abstract}
Background. Burns are common traumas that cause systemic symptoms by increasing vascular permeability.

Objectives. To investigate the role of miRNA-451 and to clarify the underlying mechanism of the burn process.

Material and methods. We established a heat-induced third-degree burn with acute lung injury (ALI) model in rats. Hematoxylin and eosin (H\&E) staining and in situ hybridization were performed. Overexpressed miRNA-451 in human umbilical vascular endothelial cells (HUVEC) were carried out. The migration and proliferation of HUVEC cells were examined.
\end{abstract}

Results. The H\&E staining showed that the burn injury caused by heat went through the dermis and damaged deep tissues. Meanwhile, the heat also induced acute lung injury, characterized by inflammatory exudation in the alveoli and significant enlargement of the alveolar septum. In situ hybridization showed that the expression of miRNA-451 increased in the lung endothelial cells. We overexpressed miRNA-451 in human umbilical vascular endothelial cells (HUVEC) and the results showed that miRNA-451 inhibited the migration and proliferation of HUVEC cells, increased HUVEC cell permeability, inhibited cell adhesion, and induced cell apoptosis. Furthermore, the expression of occludin and Z0-1, 2 key protein molecules in forming tight junction between cells, decreased, and the proteins dispersed in the cytoplasm of HUVEC cells.

Conclusions. MiRNA-451 was upregulated in the lung endothelial cells of the rat model, and contributed to increase lung endothelial cell permeability. It suppresses angiogenesis of lung endothelial cells, indicating their potential as a target in the treatment of burn injuries.

Key words: angiogenesis, burn, endothelial cell, miRNA-451, hyperpermeability

Cite as

Zhou J, Lian H, Xu G, Zhao T. MicroRNA-451 increases vascular permeability and suppresses angiogenesis in pulmonary burn injury in a rat model. Adv Clin Exp Med. 2020;29(11):1241-1248. doi:10.17219/acem/126299

DOI

10.17219/acem/126299

Copyright

Copyright by Author(s)

This is an article distributed under the terms of the

Creative Commons Attribution 3.0 Unported (CC BY 3.0)

(https://creativecommons.org/licenses/by/3.0/) 


\section{Introduction}

A burn is a type of injury caused by heat, cold, electricity, chemicals, or radiation to the skin or other tissues. ${ }^{1}$ According to the severity of damage to the skin and tissues, burns are divided into 4 types: first-, second-, third-, and fourth-degree burns. One of the physiological characteristics of burns is increased vascular permeability associated with massive release of inflammatory mediators, resulting in immediate and continuous loss of substances ranging from water to macromolecules. ${ }^{2}$ This leads to the reduction of blood volume and insufficient blood supply to important organs, such as heart, brain and kidneys. Severe burn is always accompanied by infection, shock and inhalation injury, which may be associated with acute lung injury (ALI). The severe stage of ALI, known as acute respiratory distress syndrome (ARDS), has a high fatality rate. It is important to investigate related molecular mechanisms of vascular hyperpermeability in burn injury in order to develop effective therapeutics.

MicroRNAs (miRNAs) are a group of small non-coding RNA molecules that function in post-transcriptional regulation of gene expression. ${ }^{3}$ Recently, the role of miRNAs in regulating the function of endothelial cells has attracted great interests. Son et al. found that murine-specific miRNA-712 is a flow-sensitive microRNA, which is upregulated by disturbed flow in arterial endothelium both in vitro and in vivo. This miRNA activated matrix metalloproteinases (MMP2 and MMP9) and induced endothelial cell inflammation and hyperpermeability. ${ }^{4}$ MiRNA-663 is an oscillatory shear (OS)-sensitive miRNA in human umbilical vein endothelial cells (HUVECs), and is involved in OS-induced cellular inflammation by mediating the expression of inflammatory genes. Oscillatory shear-induced monocyte adhesion was blocked by treating HUVECs with miRNA-663 inhibitor. ${ }^{5}$ Thus, miRNAs are potential targets for the treatment of vascular dysfunctions during the burn process.

In this study, we established a heat-induced third-degree burn model in rats and detected the expression of miRNA-451 in lung endothelial cells. In order to discover the effects of miRNA-451 on the permeability and angiogenesis of vascular endothelial cells, we upregulated and downregulated miRNA-451 through transfecting miRNA-451 mimic or inhibitor into HUVECs. Phenotypic change of cells, including cell proliferation, cell adhesion, cell migration, and cell apoptosis, in different groups of HUVECs cultured with or without serum from rats in different burn groups were examined. Moreover, we evaluated the change of expression of $\mathrm{ZO}-1$ and occludin, which are very common tight junction associated proteins. Damaged tight junctions will cause increased permeability. $^{6}$

\section{Material and methods}

\section{Animals and groupings}

The animal experiments were conducted according to the protocols approved by the Animal Experimental Ethics Committee of the Second Affiliated Hospital of Soochow University, China. Healthy male adult Sprague-Dawley (SD) rats were purchased from Shanghai SLCA Laboratory Animal Ltd. (Shanghai, China). The weight of rats ranged from $300 \mathrm{~g}$ to $350 \mathrm{~g}$. Eighteen rats were randomly separated into 3 groups: burn group, sham burn group and control group, with 6 rats in each group. The back hair of the rats in the burn group and sham burn group were shaved under anesthesia (urethane, $5 \mathrm{mg} / \mathrm{kg}$ body weight). Then, the nude skin of the burn group was scalded with $95^{\circ} \mathrm{C}$ hot water for $18 \mathrm{~s}$, which caused a third-degree burn consisting nearly $30 \%$ of total body surface area (TBSA), while the nude skin of sham burn group was exposed to $37^{\circ} \mathrm{C}$ water for the same time. After that, the rats from the burn group were injected with physiological saline solution following the Parkland formula $(4 \mathrm{~mL} / \mathrm{kg} / 1 \%$ TBSA). All the rats were kept in individual cages and allowed free access to food and water.

\section{Hematoxylin and eosin staining}

Skin and lung samples were collected $12 \mathrm{~h}$ after burn and fixed with $4 \%$ neutral-buffered formalin for $24 \mathrm{~h}$. After dehydration and embedding, the samples were cut into sections of $3 \mu \mathrm{m}$ in thickness. The sections were stained with hematoxylin and eosin (H\&E).

\section{Colloid osmotic pressure measurements}

The plasma samples from each group were collected at different time points: $60 \mathrm{~min}$ before burn, and $0 \mathrm{~min}$, $30 \mathrm{~min}, 60 \mathrm{~min}, 90 \mathrm{~min}, 120 \mathrm{~min}$, and $180 \mathrm{~min}$ after burn. Colloid osmotic pressure was analyzed using a micro-colloid osmometer (Wescor 4420; Wescor, Logan, USA) with a semipermeable membrane, which had a rejection characteristic of $30 \mathrm{kDa}$. One to $1.5 \mu \mathrm{L}$ plasma was applied to the semipermeable membrane in each measurement. All the measurements were performed at room temperature.

\section{Cell culture and grouping}

The HUVECs were purchased from American Type Culture Collection (ATCC; Manassas, USA) and cultured in the EGM02 medium (CC03162; Lonza, Cologne, Germany). MiRNA-451 mimic and inhibitor (GenePharma, Shanghai, China) were transfected into the cells using Lipofectamine 2000 (11668027; Thermo Fisher Scientific, Waltham, USA). Then, the HUVECs were divided into 7 groups:

- group 1: cells cultured with medium supplemented with 10\% FCS (Gibco, Rockville, USA); 
- group 2: cells cultured with medium supplemented with $10 \%$ rat serum from burn group;

- group 3: cells cultured with medium supplemented with $10 \%$ rat serum from control group;

- group 4: cells transfected with miRNA-451 mimic and cultured with medium supplemented with $10 \%$ rat serum from burn group;

- group 5: cells transfected with scramble control for miRNA-451 mimic and cultured with medium supplemented with $10 \%$ rat serum from burn group;

- group 6: cells transfected with miRNA-451 inhibitor and cultured with medium supplemented with $10 \%$ rat serum from burn group;

- group 7: cells transfected with scramble control for miRNA-451 inhibitor and cultured with medium supplemented with $10 \%$ rat serum from burn group.

\section{Real-time PCR}

The expression of miRNA-451 in the HUVECs after transfection was examined using real-time polymerase chain reaction (RT-PCR). The total RNA of cells was isolated by lysing the cells with Trizol (15596018; Thermo Fisher Scientific) and reversed transcribed into cDNA. Realtime PCR was carried out using $2 \times$ SYBR Premix Ex Taq (RR820A; Takara Bio, Shiga, Japan) according to the manufacturer's protocol. Primers were purchased from Sangon Biotech (Shanghai, China) as follow: forward: 5'-ACACTCCAGCTGGGAAACCGTTACCATTAC, reverse: 5'TGGTGTCGTGGAGTCG. The relative gene expression was analyzed using the $2^{-\Delta \Delta \mathrm{CT}}$ method.

\section{Cell proliferation}

Cell counting kit-8 assay (CCK-8; Dojindo, Kumamoto, Japan) was used to detect the proliferation of the HUVECs from each group. According to the manufacturer's protocol, $5 \times 10^{3}$ cells/well were seeded into 24 -well plate and culture for $72 \mathrm{~h}$. After culturing, CCK8 solution was added into each well and the light absorbance of each well was measured at $450 \mathrm{~nm}$ using a microplate reader (Multiskan FC; Thermo Fisher Scientific).

\section{Cell apoptosis}

Annexin V/PI stain and flow cytometer were used for detecting cell apoptosis. In brief, HUVECs from each group were suspended in binding buffer at a concentration of $3 \times 10^{5}$ and incubated with Annexin V and PI for $15 \mathrm{~min}$ at room temperature in the dark. Then the cells were examined with a flow cytometer (LSRFortessa; Becton Dickinson, Franklin Lakes, USA) and the results were analyzed with FlowJo software v. 7.6.1 (www.flowjo.com). The annexin V+/PI- cells and annexin V-/PI+ cells were used to identify apoptotic cells and necrotic cells, respectively. The procedure was repeated 3 times for each sample.

\section{Scratch assay}

The HUVECs from each group were culture in six-well plates and wounds were made using a $200 \mu \mathrm{L}$ tip when cell monolayers were formed. After cell debris was removed, the cells were cultured with serum-free medium, and the wounds were observed and captured at $24 \mathrm{~h}$ and 48 h using an inverted light microscope (Olympus BX43; Olympus, Tokyo, Japan). Three independent experiments were performed in each group.

\section{Cell permeability}

In vitro cell permeability assay was performed as previously described. ${ }^{7}$ In brief, HUVECs from each group were seeded into type I collagen-coated upper chambers ( $0.4 \mu \mathrm{m}$ pores; Becton Dickinson) and were grown to confluence. Fifteen microliters of fluorescein isothiocyanate (FITC)-dextran (Sigma-Aldrich, St. Louis, USA) was added to the upper chamber and incubated at $37^{\circ} \mathrm{C}$ for $30 \mathrm{~min}$. Then, fluorescence intensity in the lower chambers, which diffused from upper chambers, was measured.

\section{Western blotting analysis}

The HUVECs from each group were lysed with radioimmunoprecipitation assay buffer (RIPA). The total proteins were loaded to SDS-PAGE gel and separated with electrophoresis. Then, the proteins were transferred to the polyvinylidene fluoride (PVDF) membrane, followed by blocking with $5 \%$ non-fat milk. After that, the membrane was blotted with the primary antibodies, including occludin (sc133256; Santa Cruz Biotechnology, Santa Cruz, USA) and ZO-1 (\#5406; Cell Signaling Technology, Danvers, USA), and secondary antibodies. Chemiluminescence was used to detect the specific bands.

\section{Statistical analyses}

All the quantitative data of each experiment were expressed as the mean \pm standard deviation (SD) and the variables were analyzed using one-way analysis of variance (ANOVA); the Tukey's method was used for the following multiple comparison tests. Apoptosis ratio data was evaluated with the $\chi^{2}$ test. SPSS software v. 20.0 (IBM Corp., Armonk, USA) was used for all the statistical analyses. A value of $\mathrm{p}<0.05$ was considered to be statistically significant.

\section{Results}

\section{Establishment of a burn model in rat}

Under a microscope, the epidermis showed nuclear condensation, and necrotic hair follicles and sebaceous glands were observed in the dermis of rats in the burn group. 
The lesions were in line with the characteristics of thirddegree burn damage. There were no significant abnormal changes in the dermis and epidermis of rats in the sham group and the control group (Fig. 1A).

It was known that local burns could lead to acute injury to the lung by inducing the release of pro-inflammatory cytokines. ${ }^{7}$ We collected the lung tissues from rats in each group and performed $H \& E$ staining. It was noted that the alveolar septum was significantly widened and there was a large number of neutrophils infiltration in the lung tissue of burned rats. There was also markedly inflammatory exudation in the alveoli, leading to the decrease of alveolar space in the burn group compared to the sham group and the control group. There was no significant abnormal change in the lung tissue of the sham group and the control group, as shown in Fig. 1B.
A
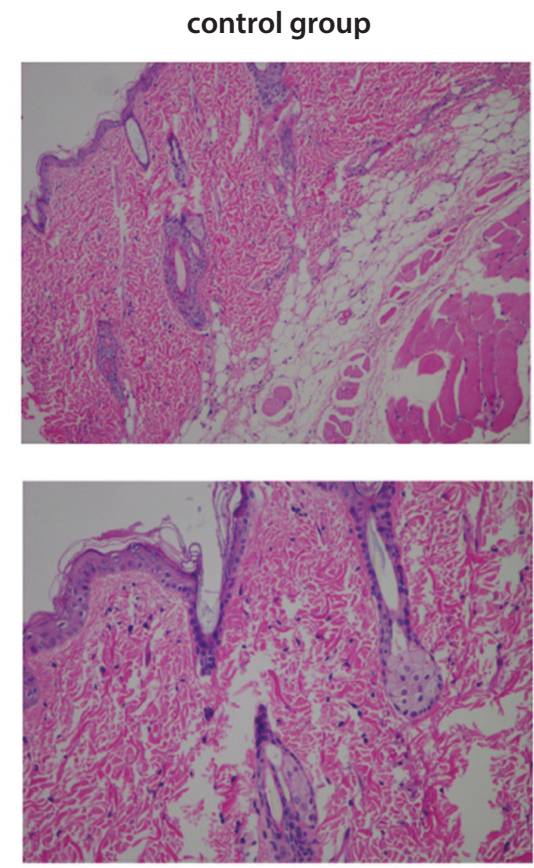

B

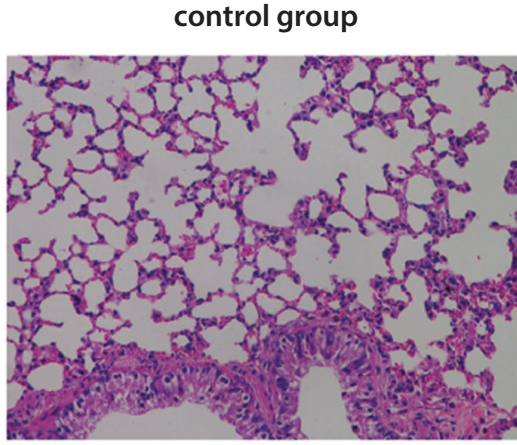

sham group
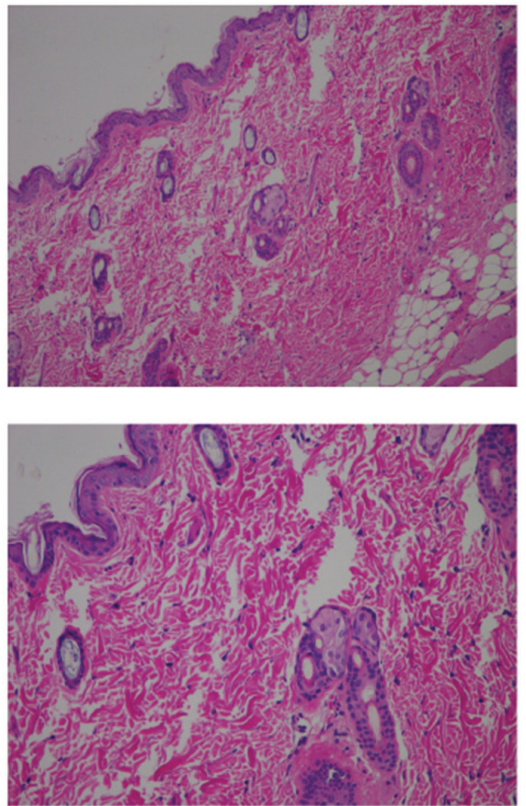

sham group

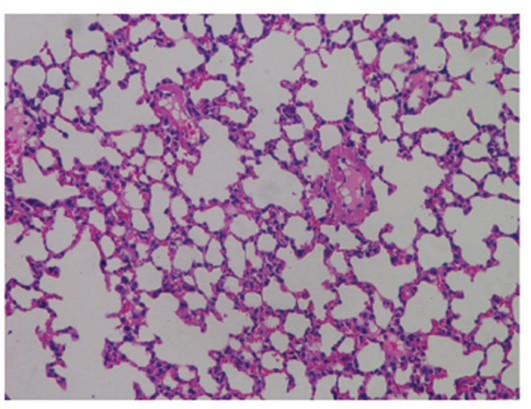

burn group
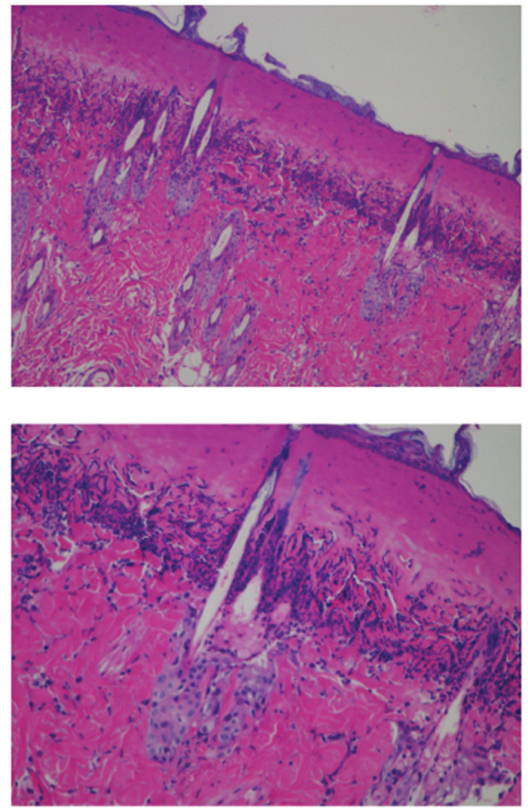

burn group

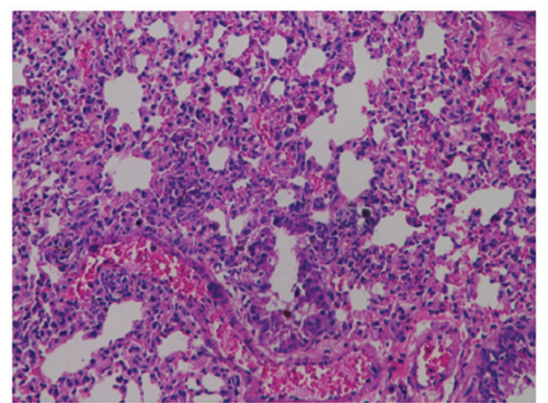

C

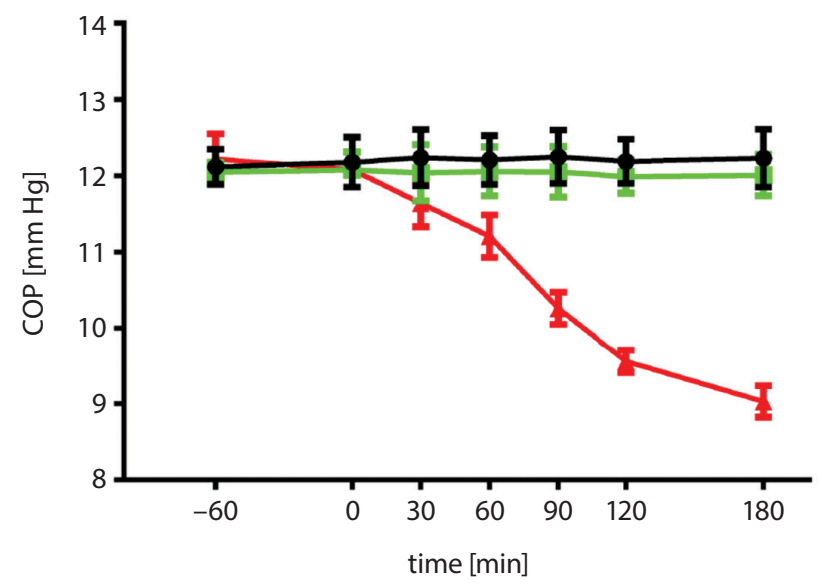

- control group

- sham group

\pm burn group
Fig. 1. Burn model of rat. A. H\&E staining of rat skin in burn group, sham group and control group $(\times 100$ and $\times 200$ magnification). B. H\&E staining of rat lung tissues in burn group, sham group and control group (×200 magnification). C. Colloid osmotic pressure in burn group, sham group and control group at different time points after the establishment of burn injury model 

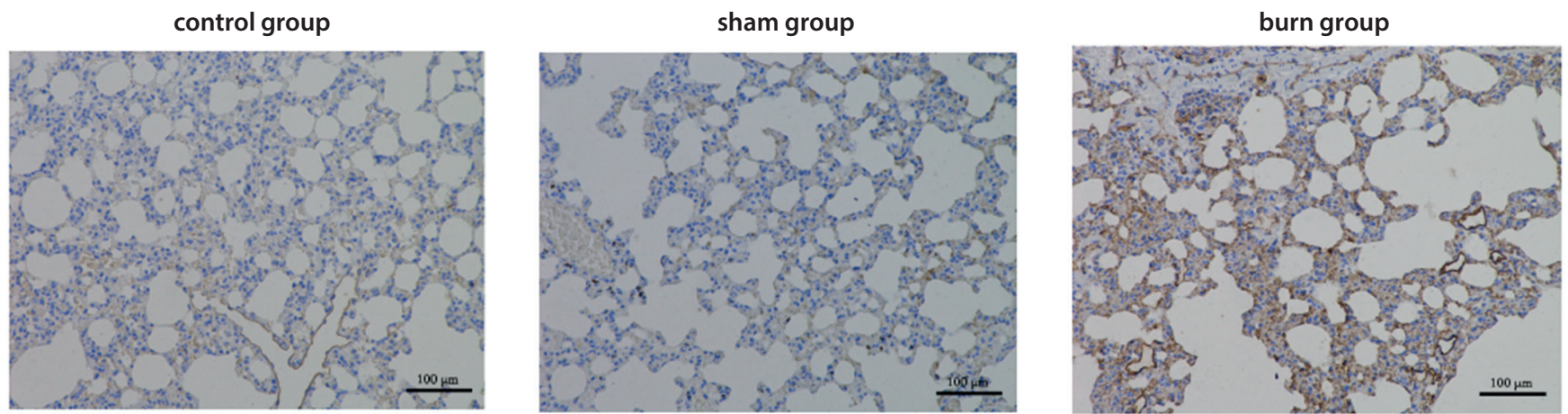

Fig. 2. MiRNA-451 upregulated in the lung microvascular endothelial cells after the establishment of burn injury. In situ hybridization on the expression of miRNA-451 in lung endothelial cell in burn group, sham group and control group (×200 magnification)

We also collected the plasma from each group at different time points to examine the variation of colloidal osmotic pressure. The results showed that colloidal osmotic pressure was continuously decreased from $12.8 \pm 0.2 \mathrm{~mm} \mathrm{Hg}$ to $9.4 \pm 0.3 \mathrm{~mm} \mathrm{Hg}$ in the burn group after the burn injury was established. However, no significant changes were found in the sham burn group and the control group (around $12.4 \mathrm{~mm} \mathrm{Hg}$ ) (Fig. 1C).

\section{Increased expression of miRNA-451 in the lung microvascular endothelial cells after burn}

It was reported that miRNA-451 was upregulated in denatured dermis of severely burned patients. ${ }^{8}$ In order to understand the potential role of miRNA-451 in the burn process, we used in situ hybridization to examine its expression of miRNA-451 in the microvascular endothelial cells from each group. It was found that the expression of miRNA-451 was significantly upregulated in the burn group compared with the sham burn group and control group (Fig. 2), indicating that miRNA-451 played a role in regulating the phenotypic and functional changes of endothelial cells during the burn process.

\section{MiRNA-451 promoted apoptosis, and inhibited proliferation and migration of endothelial cells}

Because miRNA-451 was upregulated in the microvascular endothelial cells in vivo, we altered the expression of miRNA-451 in HUVECs by transfecting its mimic or inhibitor, and treating the cells with serum separated from the rats of the burn group in order to further clarify its effect on endothelial cells. The RT-PCR results showed that the expression of miRNA-451 increased in the group transfected with mimic $(2.86 \pm 0.27)$ and decreased in the group transfected with inhibitor $(0.26 \pm 0.06)$ (Fig. 3A).

We further used CCK8 assay to detect cell proliferation. The results showed that HUVECs treated with the serum from the rats of the burn groups displayed a significant decrease in cell growth when compared to the same cells treated with the serum separated from the control group, which was $1.353 \pm 0.09$ and $1.564 \pm 0.12$, respectively. Importantly, miRNA-451 mimics could work in concert with the serum separated from the rats of the burn group to inhibit the cell proliferation; the result of optical density (OD) $450 \mathrm{~nm}$ was $0.576 \pm 0.07$. Furthermore, miRNA-451 inhibitor induced the cell growth in HUVECs $(2.011 \pm 0.11)$, indicating that miRNA-451 inhibited cell proliferation in burn process (Fig. 3B).

Fluorescence-activated cell sorting (FACS) and scratch assay were used to detect cell apoptosis and cell migration in each group. PI/Annexin V double staining showed that more apoptotic cells were readily seen in the HUVECs transfected with miRNA-451 mimics and treated with the serum separated from the rats of burn group, indicating that miRNA-451 could induce apoptosis in the burn process (Fig. 3C). Meanwhile, miRNA-451 also displayed its ability to inhibit cell migration. The apoptosis rate of group 4 (cells transfected with miRNA-451 mimic and cultured with medium supplemented with $10 \%$ rat serum from burn group) was dramatically higher than in group 6 (cells transfected with miRNA-451 inhibitor and cultured with medium supplemented with $10 \%$ rat serum from the burn group) (21.35 \pm 0.93 and $2.37 \pm 0.38)$ (Fig. 3D).

\section{MiRNA-451 increased the permeability of HUVEC cells}

Hyperpermeability occurs in vascular endothelial cells during the burn process. Cell permeability was evaluated in HUVECs under different conditions. The results showed that upregulation of miRNA-451 in HUVECs displayed an increase in permeability, since the intensity of FITCdextran increased in the bottom cell culture chamber, which was $55.4 \pm 6.3 \%$. The opposite effects were found in the HUVECs with the downregulation of miRNA-451 cells $(10.35 \pm 2.43 \%)$ (Fig. $4 \mathrm{~A})$.

The results of western blot analysis showed that the serum separated from the rats of the burn group downregulated the expression of occludin and ZO-1; the effects 

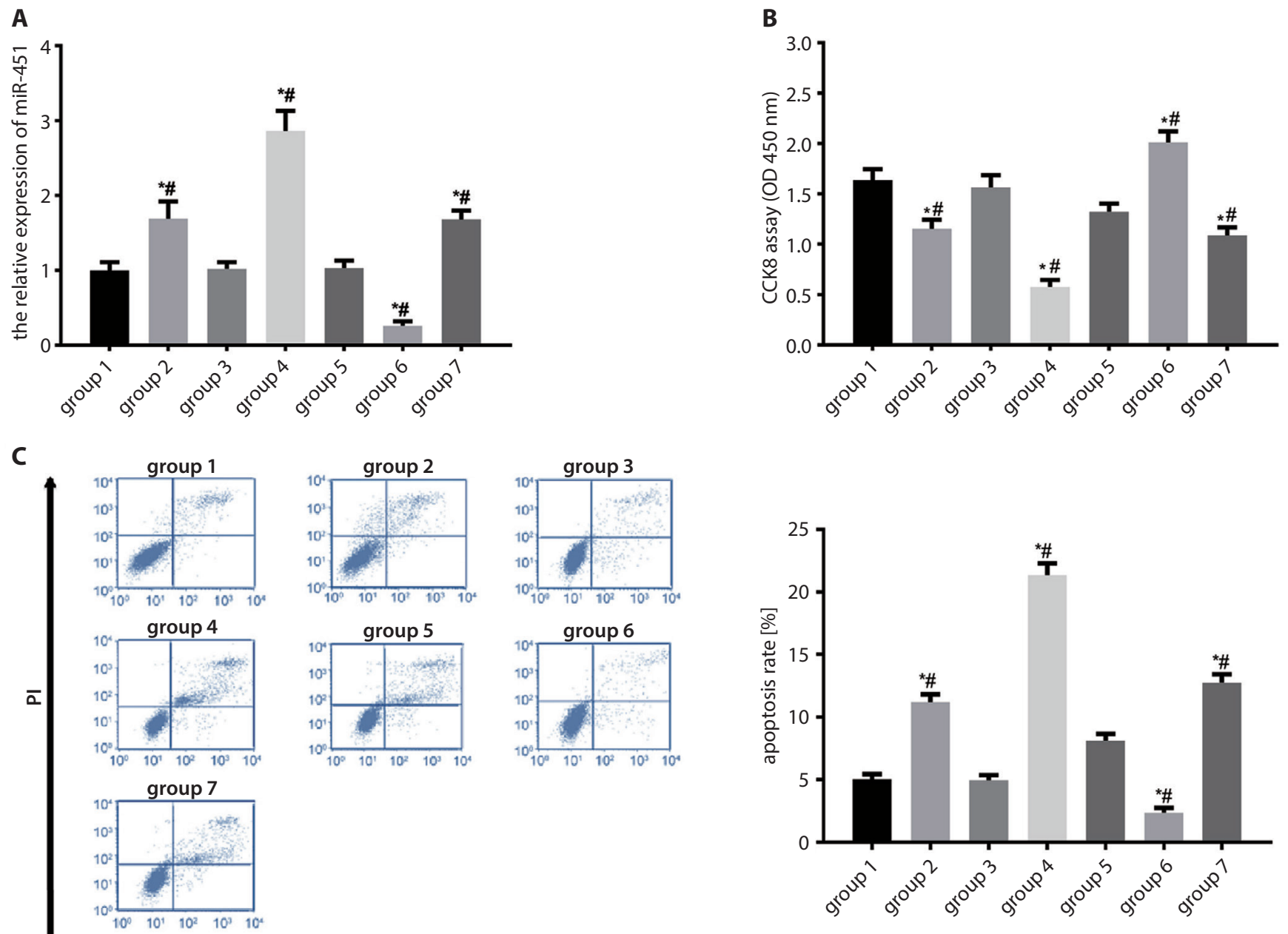

annexin V-FITC

D
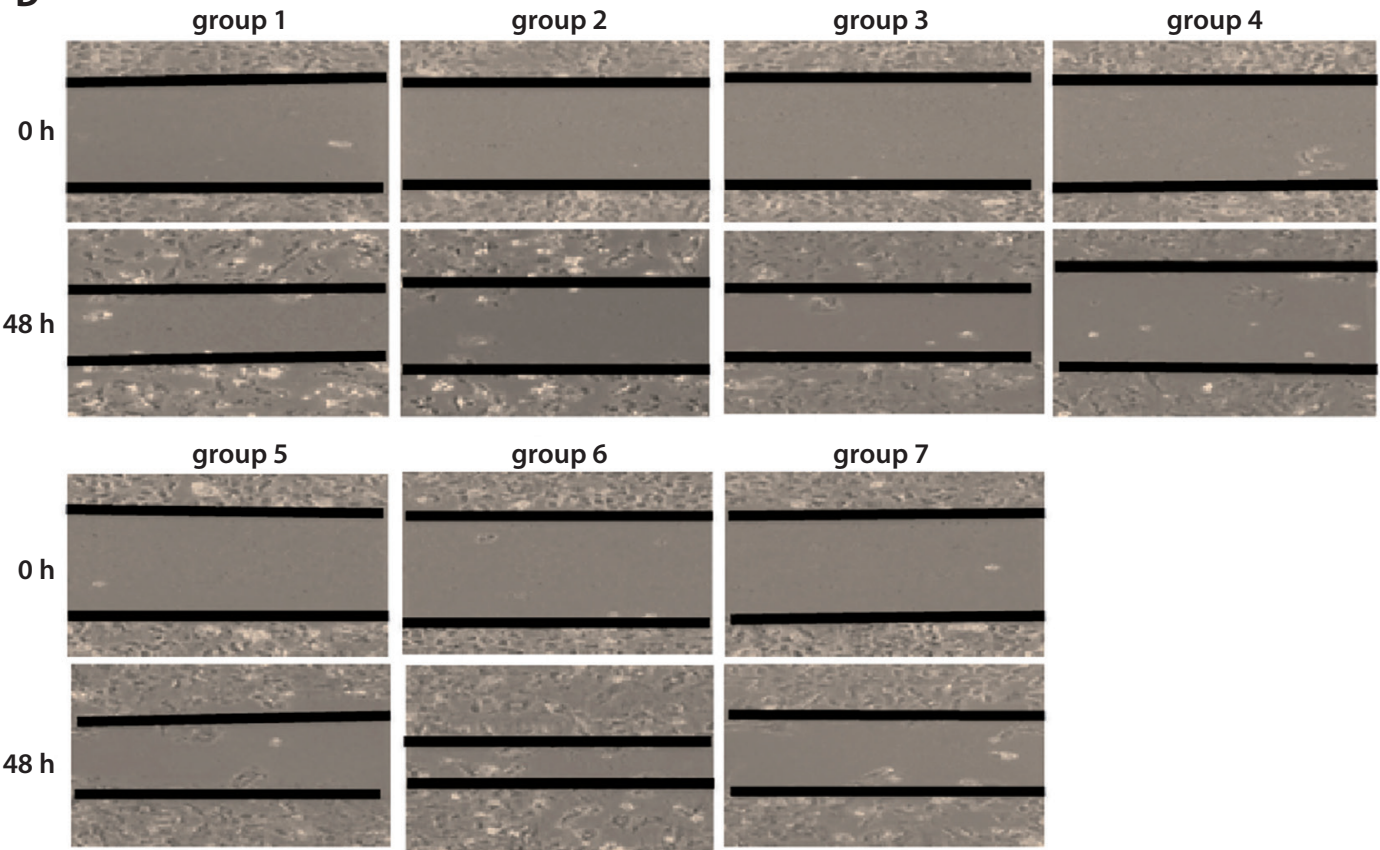

Fig. 3. MiRNA-451 regulated the proliferation, apoptosis and migration of HUVECS. A. RT-PCR on the expression of miRNA-451 expression in HUVECS in different groups after transfection. B. CCK8 assay on the cell proliferation in various groups. C. FACS on the cell apoptosis in various groups. D. Scratch assay on the cell migration in various groups

Compared with control group, ${ }^{*} p<0.05$. Compared with sham burn group, $\# p<0.05$. 
A

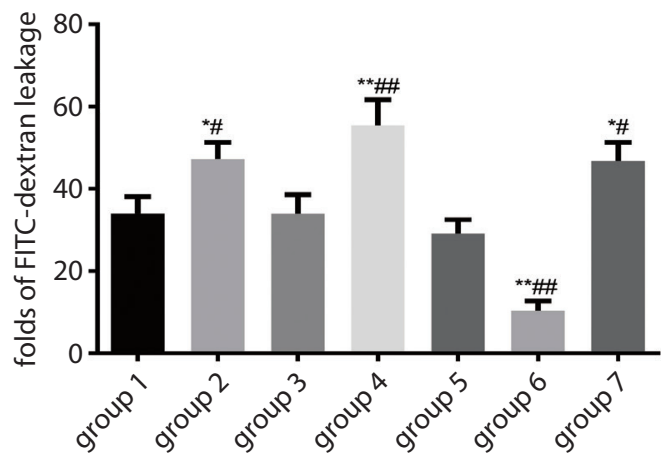

B

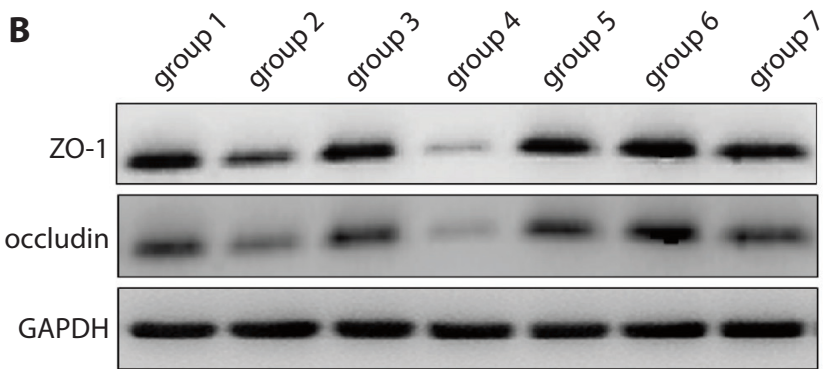

Fig. 4. MiRNA-451 increased the permeability of HUVECs. A. The HUVEC cell permeability in various groups. B. Western blot analysis on the expression of ZO-1 and occludin in HUVECs in various groups

were more obvious in the HUVECs transfected with miRNA-451 mimics. The miRNA-451 inhibitor antagonized the effects caused by the serum separated from the rats of burn group, since the expression of occludin and ZO-1 was not downregulated (Fig. 4B).

\section{Discussion}

Vascular hyperpermeability is a critical issue in the burn process, since it results in the release of a number of pro-inflammatory cytokines, including interleukin 1 beta (IL-1 $\beta$ ), tumor necrosis factor $\alpha$ (TNF- $\alpha)$ and other inflammatory factors, ${ }^{9}$ leading to systemic symptoms. However, the mechanisms of vascular hyperpermeability in burn injury are not completely understood. Recently, the role of miRNAs in regulating the functions of endothelial cells has attracted great interests. ${ }^{10}$ In the present study, a thirddegree burn model in rats was established. The H\&E staining showed that the burn injury went through the dermis and affected deep tissues. Furthermore, the burns on the back skin of rats resulted in ALI, indicating the release of pro-inflammatory factors and the injury of functions of endothelial cells.

In situ hybridization (ISH) is a mature methodology for assessing miRNA expression. ${ }^{11-13}$ It could detect specific nucleic acid sequences in cellular level in tissue samples. The ISH technology could provide more information about the cellular origin of expression and on expression levels in different tissue compartments and cell populations, aiding in the detection of individual miRNAs and mRNAs. ${ }^{12}$
The results of ISH showed the overexpression of miRNA-451 in the endothelial cells of burn group, indicating that miRNA-451 played a role in regulating the phenotypic and functional changes of endothelial cells during the burn process. Moreover, this result is consistent with the previous report that miRNA-451 was upregulated in denatured dermis of severely burned patients, ${ }^{8}$ demonstrating that miRNA-451 is a factor regulating the permeability of endothelial cells in the burn process.

Cell-cell junctions that provide structural integrity are essential elements in maintaining homeostasis in endothelial cells. ${ }^{14}$ In particular, tight junctions control the permeability in endothelial cells. ${ }^{15}$ Occludin, a transmembrane protein, is one of the key components of tight junctions which regulates actin assembly. ${ }^{16} \mathrm{ZO}-1$ is a protein which plays a role in anchoring occludin to the actin assembly. ${ }^{17}$ Thus, occludin along with ZO-1 maintains the integrity and barrier functions of endothelia. However, burns damage the inter-endothelial junctions ${ }^{18}$ and lead to the leakage of substances ranging from water to macromolecules. In our study, we found that the serum from the rats of the burn group decreased the expression of ZO-1 and occludin, and the effects were simulated by miRNA-451 mimics in HUVECs, leading to microvascular hyperpermeability. On the other hand, downregulation of miRNA-451 antagonizes the effects caused by the serum from the burn group.

Angiogenesis is a biological mechanism of new capillary formation which supplies oxygen and nutrients to cells in the wound area and regenerates tissues. ${ }^{19}$ Angiogenesis is critical in determining the wound-healing outcomes in third-degree burns. ${ }^{20}$ Recently, the correlation between miRNAs and angiogenesis has been studied. Soufi-Zomorrod used bioinformatics algorithms to identify that VEGFR2 and FGFR1 were the targets of miRNA-129-1 and miRNA-133. The HUVECs transfected with miRNA-129-1 and miRNA-133 mimics suppressed the angiogenesis properties, including cell proliferation, cell viability and migration. ${ }^{21}$ Liu reported that the elevation of miRNA-451 in human hepatocellular carcinoma (HCC) cells mitigated the viability, migration and tube formation of HUVECs by targeting IL-6-STAT3VEGF signaling. Its overexpression also reduced angiogenesis of HCC cells in vivo. ${ }^{22}$ In our study, we transfected HUVECs with miRNA-451 mimic and inhibitor, and the results showed that miRNA-451 mimics could suppress the cell proliferation and migration and induce apoptosis in HUVECs, and the effects were similar to those of the treatment with serum from rats with third-degree burns, indicating that miRNA-451 is a key regulator inhibiting angiogenesis in burn process. Li Q et al. study also shown that miRNA-451 had anti-angiogenic effect, and downregulation of miRNA-451 would promote the migration of HUVEC. ${ }^{23}$

In summary, this study showed that miRNA-451 was overexpressed in lung endothelial cells in rats with 
third-degree burns. In vitro experiments suggested that miRNA-451 may play a role in suppressing angiogenesis and increasing permeability of HUVECs. MiRNA-451 could be a potential target for the treatment of burn injury.

\section{ORCID iDs}

Jie Zhou (D) https://orcid.org/0000-0002-9217-9516

Huibin Lian (D) https://orcid.org/0000-0003-2846-2779

Tianlan Zhao (i) https://orcid.org/0000-0002-9146-2782

Gang Xu (1) https://orcid.org/0000-0002-9186-7173

\section{References}

1. Herndon DN. Total Burn Care. $4^{\text {th }}$ ed. New York, NY: Saunders Elsevier 2012.

2. Turk E, Caliskan M, Karagulle E, et al. A prospective clinical study of flow-mediated dilatation in burn injury. J Burn Care Res. 2014;35(2): 169-175.

3. Bartel DP. MicroRNAs: Genomics, biogenesis, mechanism, and function. Cell. 2004;116(2):281-297.

4. Son DJ, Kumar S, Takabe W, et al. The atypical mechanosensitive microRNA-712 derived from pre-ribosomal RNA induces endothelial inflammation and atherosclerosis. Nat Commun. 2013;4:3000.

5. Ni CW, Qiu H, Jo H. MicroRNA-663 upregulated by oscillatory shear stress plays a role in inflammatory response of endothelial cells. Am J Physiol Heart Circ Physiol. 2011;300(5):H1762-H1769.

6. Bazzoni G, Martinez-Estrada OM, Orsenigo F, Cordenonsi M, Citi S, Dejana E. Interaction of junctional adhesion molecule with the tight junction components ZO-1, cingulin, and occludin. J Biol Chem. 2000; 275(27):20520-20526.

7. Chen F, Ohashi N, Li W, Eckman C, Nguyen JH. Disruptions of occludin and claudin-5 in brain endothelial cells in vitro and in brains of mice with acute liver failure. Hepatology. 2009:50(6):1914-1923.

8. Liang $P, L v C$, Jiang $B$, et al. MicroRNA profiling in denatured dermis of deep burn patients. Burns. 2012;38(4):534-540.

9. Ravat F, Payre J, Peslages P, Fontaine M, Sens N. Burn: An inflammatory process [in French]. Pathol Biol (Paris). 2011;59(3):e63-e72.
10. Cheng XW, Wan YF, Zhou Q, Wang Y, Zhu HQ. MicroRNA-126 inhibits endothelial permeability and apoptosis in apolipoprotein E-knockout mice fed a high-fat diet. Mol Med Rep. 2017;16(3):3061-3068.

11. Nelson PT, Wilfred BR. In situ hybridization is a necessary experimental complement to microRNA (miRNA) expression profiling in the human brain. Neurosci Lett. 2009;466(2):69-72.

12. Nielsen BS. MicroRNA in situ hybridization. Methods Mol Biol. 2012; 822:67-84.

13. Li J, Li X, Li Y, et al. Cell-specific detection of miR-375 downregulation for predicting the prognosis of esophageal squamous cell carcinoma by miRNA in situ hybridization. PLoS One. 2013;8(1):e53582.

14. Dejana E, Tournier-Lasserve E, Weinstein BM. The control of vascular integrity by endothelial cell junctions: Molecular basis and pathological implications. Dev Cell. 2009:16(2):209-221.

15. Steed E, Balda MS, Matter K. Dynamics and functions of tight junctions. Trends Cell Biol. 2010;20(3):142-149.

16. Kuwabara H, Kokai Y, Kojima T, Takakuwa R, Mori M, Sawada N. Occludin regulates actin cytoskeleton in endothelial cells. Cell Struct Funct. 2001;26(2):109-116.

17. Van Itallie CM, Tietgens AJ, Krystofiak E, Kachar B, Anderson JM. A complex of ZO-1 and the BAR-domain protein TOCA-1 regulates actin assembly at the tight junction. Mol Biol Cell. 2015;26(15):2769-2787.

18. Wiggins-Dohlvik K, Suk Han M, Stagg HW, Alluri H. Melatonin inhibits thermal injury-induced hyperpermeability in microvascular endothelial cells. J Trauma Acute Care Surg. 2014;77(6):899-905, discussion 905.

19. Gonzalez AC, Fortuna Costa T, de Araújo Andrade Z, Ribeiro Alves Peixoto Medrado A. Wound healing: A literature review. An Bras Dermatol. 2016;91(5):614-620.

20. Tredget EE. The basis of fibrosis and wound healing disorders following thermal injury. J Trauma. 2007;62(6 Suppl):S69.

21. Soufi-Zomorrod M, Hajifathali A, Kouhkan F, Mehdizadeh M, Hosseini Rad SMA, Soleimani M. MicroRNAs modulating angiogenesis: miR-129-1 and miR-133 act as angio-miR in HUVECs. Tumor Biol. 2016; 37(7):9527-9534

22. Liu XM, Zhang A, Xiang J, Lv Y, Zhang X. miR-451 acts as a suppressor of angiogenesis in hepatocellular carcinoma by targeting the IL-6RSTAT3 pathway. Oncol Rep. 2016;36(3):1385-1392.

23. Li Q, Zhang D, Gao H, Gao X. Downregulation of microRNA-451 improves cell migration, invasion and tube formation in hypoxiatreated HUVECs by targeting MIF. Mol Med Rep. 2019;20:1167-1177. 